\title{
Product Lifecycle Management Adoption versus Lifecycle Orientation: Evidences from Italian Companies
}

\author{
Monica Rossi ${ }^{1}$, Dario Riboldi ${ }^{1}$, Daniele Cerri ${ }^{1}$, Sergio Terzi ${ }^{2}$, and Marco Garetti ${ }^{1}$ \\ ${ }^{1}$ Politecnico di Milano, Milan, Italy \\ ${ }^{2}$ Università degli Studi di Bergamo, Bergamo, Italy \\ \{monica.rossi, daniele.cerri, marco.garetti\}@polimi.it, \\ dario.riboldi@mail.polimi.it, \\ sergio.terzi@unibg.it
}

\begin{abstract}
The present paper shows some of the results coming from an Italian research initiative in the field of PLM. Aim of this initiative - partly supported by the Linked Design European project - is to conduct an empirical research on the main drivers and issues related with the implementation of PLM in industries. In particular, the relationship between "PLM adopter" and "lifecycle-oriented" companies is discussed in this paper.
\end{abstract}

Keywords: Product Lifecycle Management, Lifecycle orientation, Empirical research.

\section{Introduction}

What is PLM (Product Lifecycle Management)? How do enterprises intend these "lifecycle issues"? Are companies buying PLM suites also becoming "lifecycleoriented"? These questions are often discussed at industrial level and some practitioners are struggling on them. The practical experience shows how PLM and its IT tools are more and more diffused in industries, even if a general confusion is still affecting the PLM concept itself. This issue is not new and it has its origin on the first days of the PLM market, when many acronyms were created to address the same issue. This confusion is in some way due to the same market opportunities that PLM has created in the last years: tons of vendors, consultants, practitioners are using the PLM acronym to present their methods, make their offers and sell their solutions, even if - at least sometimes - they support just a small slice of "lifecycle".

Within PLM, the term lifecycle has a central role, not only from a mere graphical point of view. Each product has a lifecycle: it is created, produced, delivered, used, maintained, retired, dismissed, etc. Several issues are pushing companies to consider the lifecycle of their products: for example, customers call for more reliable solutions, as well as the society asks for greener and less-polluting systems, while regulations demand to track all the history of an equipment, etc. In this, ideally, PLM aims to track, trace, store, manage, share all the knowledge generated along an artifact lifecycle, from its creation, design, development, to its production, distribution, till its 
utilization, dismantling, etc. In the real world, so far PLM has been limited to activities related with the design and development of a product, generally realized along the innovation process of manufacturing companies. In some way, it is possible to observe how the "lifecycle management concept" is still far from the real application of "PLM systems". Aim of this paper is exactly to arise this issue: are companies adopting PLM also lifecycle-oriented?

This paper comes from a research initiative currently on going in Italy, the socalled GeCo Observatory, which is collecting industrial experiences and case studies related to the PLM concept. The GeCo Observatory also works within the wider context of a European project, Linked Design, which aims to create the next generation of PLM solutions. The paper is organized as follow: section 2 gives an overview on the PLM concept, section 3 shows the results of the empirical research done in the Italian market, while section 4 debates the main results and concludes the paper.

\section{Definition of PLM}

Different issues contribute to the definition of PLM. First, PLM is the activity of managing product across the lifecycle, a kind of new model for managing company's processes in a more integrated and efficient lifecycle-oriented way [5]. For supporting this lifecycle perspective, information management is mandatory: PLM aims to post the right information, at the right moment, in the right place for an efficient capitalization of intellectual capital of a company [1]. Comprehensively, PLM is a business concept that leads the usage of all the information connected to the product in the organization [6]. Because of this holistic nature, the concept of PLM in an organization comprehends also IT aspects of it. Although IT is not sufficient to describe the entire concept, PLM is mainly based on IT implementations for the integration of people, processes, data, and information. In its IT essence, PLM is an extension of old PDM (Product Data Management) platforms that try to overcome the PDM boundaries, from the management of design data to - ideally - the planning and control of all the processes along the whole lifecycle. PLM IT systems have the purpose to connect engineering and design software to the other suites of manufacturing and supply chain in the organization [2] and generate a common view for all the product stakeholders.

As it is known, in the market there is thousands of IT solutions tagged as "PLMready", from CAD systems to web-based vaults. In the last year, PLM acronym has been added to the catalogue of many vendors, selling FEM solutions as well ERP suites. Today, the PLM IT market is made by a plethora of providers and many companies are nowadays adopting a PLM IT system, or part of it, in their business. But are these "PLM adopters" also "lifecycle-oriented companies"? Does really the PLM concept come first than the PLM IT system? These shiny questions arose in the mind of the authors and some answers are presented in the next paragraph. 


\section{Empirical Research}

This research is part of a broader empirical study conducted by the researchers of the GeCo Observatory, an Italian initiative supported also by the Linked Design European project. The main research aims at investigating how manufacturing companies are structuring and managing their product design, development and engineering processes. The research is conducted with direct interviews made with technical directors, using a reference semi-structured questionnaire. This paper refers specifically to the questions and the collected data dealing with the company lifecycle consciousness and the adoption of PLM tools. 103 companies, coming from different sectors, compose the analyzed sample for this paper. 49 of them are typical Small and Medium Enterprises (SMEs), while 54 are bigger companies. The reference questionnaire adopted in the research is based on a CMMI maturity model [4], which describes and analyzes the design and engineering processes of a company according to three main dimensions (Organization, Process and Knowledge Management). Each dimension is divided in sub-dimensions. Each dimension is measured in terms of maturity of a specific practice, from a minimum value to a maximum one. The list of practices and their maturity have been created according to the literature, but for the purposes of this paper, it is not here detailed (see [4] for details). A subset of questions chosen from the main questionnaire was coupled to better represent the existence of a company attitude towards lifecycle consciousness (lifecycle-oriented), and the presence of PLM systems (PLM-adopter). These two dimensions are analyzed in detail in the next sections.

\subsection{Lifecycle Orientation}

The first dimension is called "Lifecycle Orientation", corresponding to the broader concept of product lifecycle introduced above. Each company provided an evaluation about how lifecycle phases are considered in taking design decisions. This evaluation is based on 5 steps, from 0 (not considered) to 4 (very high consideration). Generally, more persons answered to this question for each case and the resulting average was used as reference.

Lifecycle is defined in 3 main phases: Beginning of Life (BOL), Middle of Life (MOL) and End of Life (EOL). Data were collected using a more detailed lifecycle model, which identifies the following sub-phases:

- BOL: (i) Design and Industrialization, (ii) Manufacturing, (iii) Assembly, (iv) Testing, (v) Packaging and Warehousing,

- MOL: (i) Delivery and Distribution, (ii) Usage by the customer, (iii) Maintenance and After-sale Services provision,

- $\quad$ EOL: (i) Check, Re-Use, Updating, Revamping, and (ii) Disassembly, Recycling and Disposal.

Figure 1 shows the Lifecycle Orientation of the sample. BOL and MOL phases are normally well considered (a part sub-phases related with logistic and distribution tasks) during the design process, while EOL phase is barely considered. 


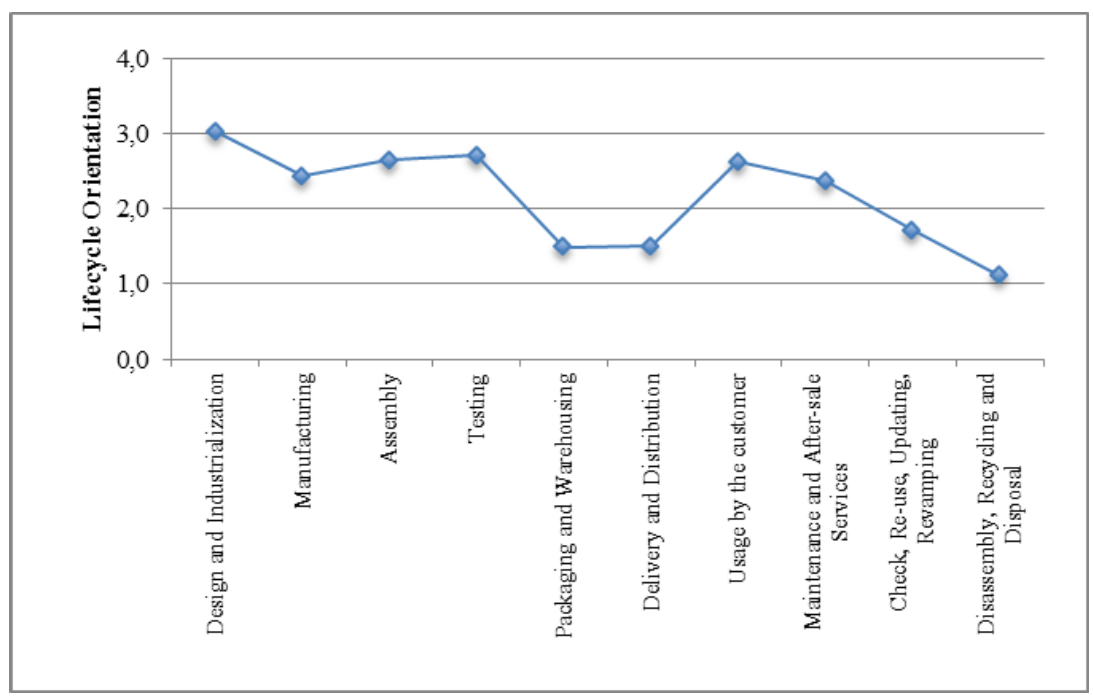

Fig. 1. Lifecycle Orientation of the sample

The degree of Lifecycle Orientation could be discussed according to two main affecting variables: (i) how the customer is involved in the design process of the company, and (ii) how the company structures itself to reply to the market demand. For the first variable, the analysis defines three positions:

- Companies not involving the customer in their design process. These companies serve the market with a detailed catalogue of solutions, not allowing relevant modifications / personalization.

- Companies involving partly representative customers in their design process, for providing them some levels of customization.

- Companies involving deeply the customer in their design process, providing highly personalized solutions.

According to this variable, Figure 2 shows that higher the possibility of product customization - consequently higher the involvement of the customer in the process higher the consciousness of the downstream phases of the process at the beginning of the lifecycle is. Generally, companies that work with the possibility of product personalization have a higher average of Lifecycle Orientation. The second variable classifies a company according to the se-called CODP (Customer Order Decoupling Point), the logical point in the development process where the possibility of a company to control the growth of the variety of finished goods ends. Generally, this point is used to classify companies according to how they respond to their customers' demand. Possible positions are: ETO (Engineering To Order), MTO (Make To 
Order), ATO (Assembly To Order), and MTS (Make To Stock). As Figure 3 shows, xTO companies (in particular ETO) have a higher consideration of downstream phases due to the customers' possibility to create their product from the upstream phases of the chain.

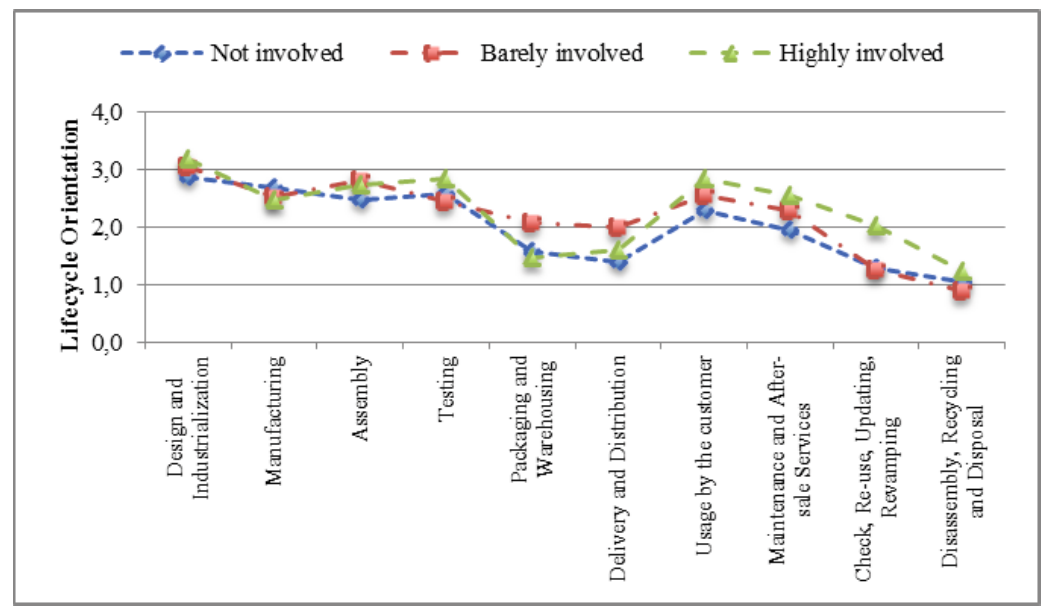

Fig. 2. Lifecycle Orientation of the sample, according to the degree of involvement of the customer in the design process

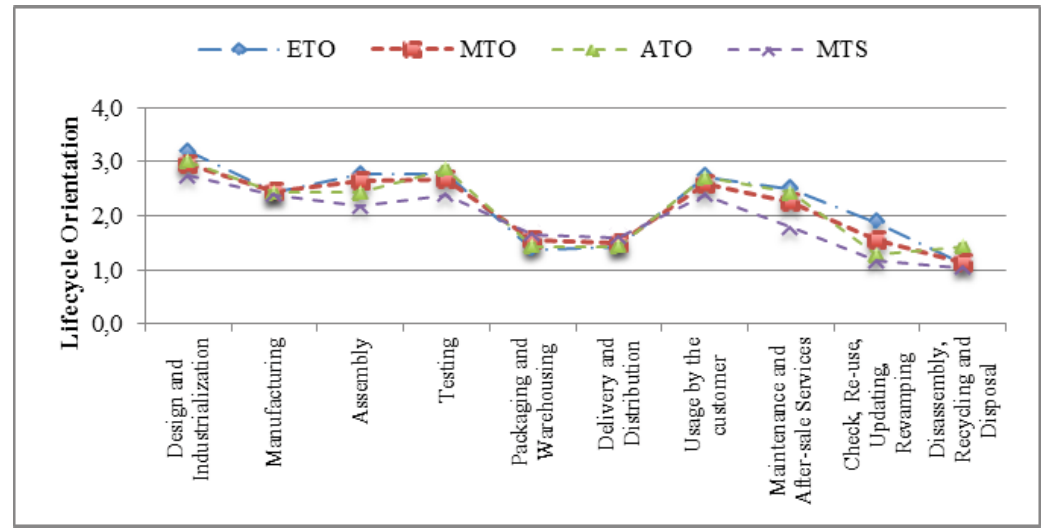

Fig. 3. Lifecycle Orientation of the sample, according to how the company replies to the market demand

\subsection{PLM Adoption}

A similar analysis has been conducted for the so-called "PLM Adoption" variable. For each single case, a deep analysis on the installed IT tools and platforms and their 
utilization has been performed. At the end, 59 companies among 103 were defined as "PLM adopters". These companies installed a PLM comprehensive platform for supporting the collaboration among different actors of the development process and they are using it for managing most of the explicit knowledge generated within.

A very interesting issue deals with the attitude of a company to reuse and retrieve knowledge from previous projects and experiences and the adoption of PLM IT systems. In the questionnaire, this analysis is made thought a complex variable which measures a maturity index (measured from 0 to 4 ): higher the index is, higher the knowledge reuse along the development process is. Figure 4 shows the results: PLM adopters re-use much more knowledge than companies without PLM. Figure 4 shows also how the index changes in the different stages of the design phase, from initial concept formulation, to detailed design, till final industrialization. This analysis confirms how PLM IT solutions are physically supporting the management of the enterprise knowledge. This happens independently from the type of industry, as well as from the type of company.

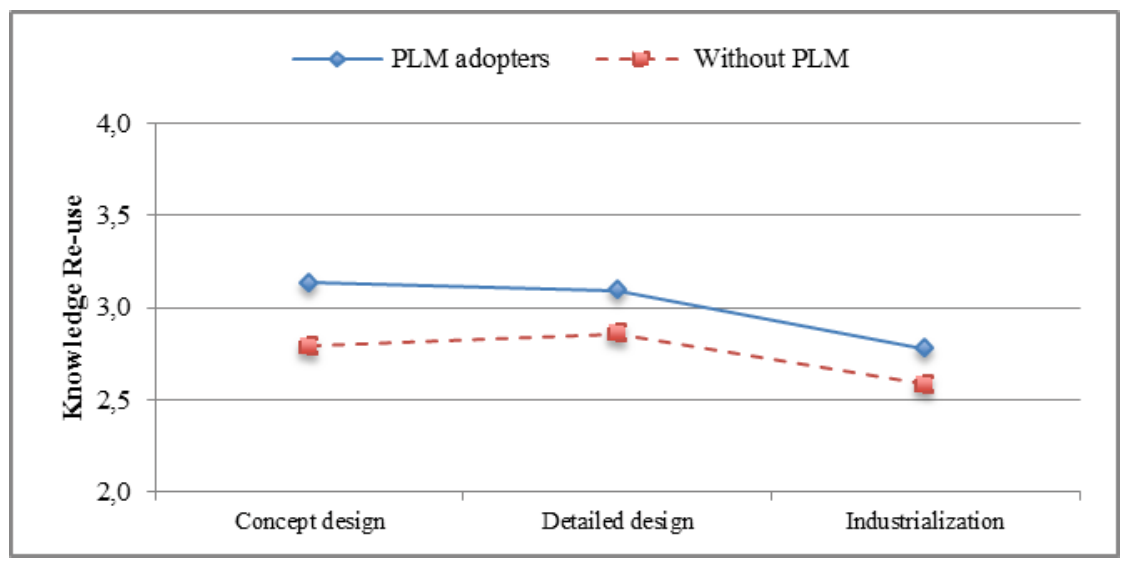

Fig. 4. Knowledge re-use versus PLM Adoption

Figure 5 represents the impact of lifecycle phases during the early stage of the process comparing PLM adopters and companies without PLM. Y-axis is the same of Figure 1, 2 and 3 (Lifecycle Orientation). It is evident how Lifecycle Orientation has a direct link with PLM Adoption. In average, PLM adopters show a higher consideration of the lifecycle, generally $15 \%$ more than companies without PLM, even if the general trend of the lifecycle consciousness of the whole sample is still confirmed. The small improvement of Lifecycle Orientation is provided by the utilization of PLM systems, which generally bring better organization and discipline in the development process of the company, supporting a more holistic view on the whole lifecycle. The data of the sample could support the evidence that companies with a high lifecycle consideration can better exploit it with a complete and conscious use of a PLM system. This evidence could be partly confirmed making a cross 
analysis of the previously presented variables. Figure 2 and 3 showed sensitively how more customer-oriented companies have higher consideration of the product lifecycle: being focused on their customers' request, they need to provide products with more reliable lifecycles. Then, Figure 6 and Figure 7 show how customer-oriented companies adopting PLM systems are more lifecycle-oriented that the same type of companies without PLM.

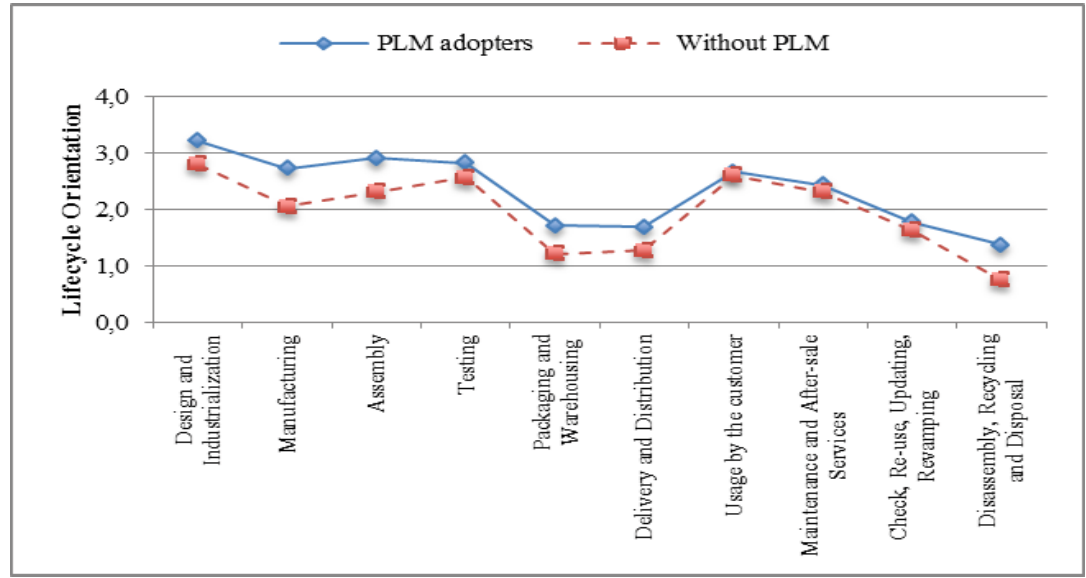

Fig. 5. Lifecycle Orientation versus PLM Adoption

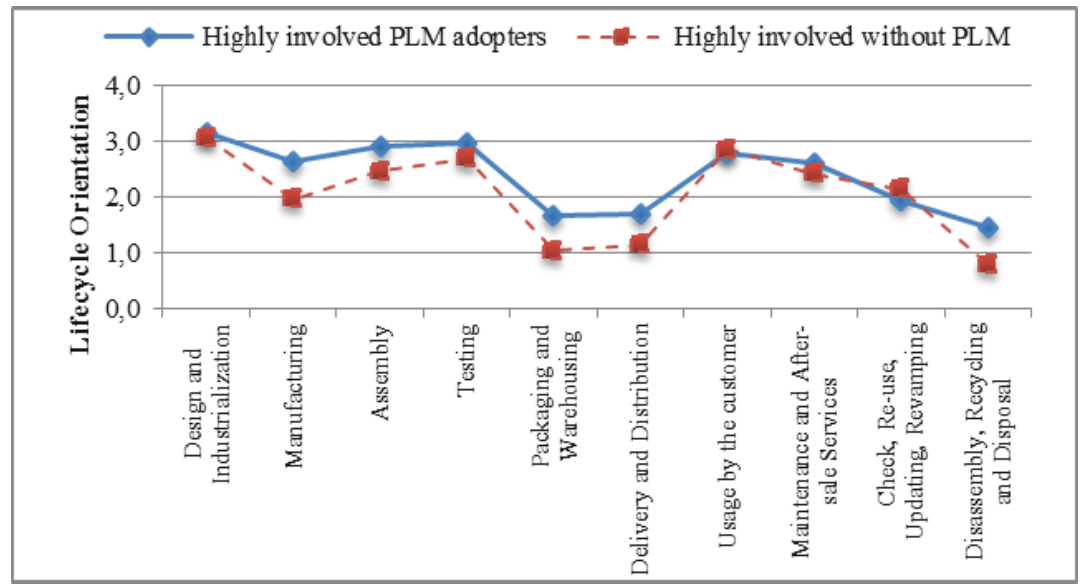

Fig. 6. Lifecycle Orientation of companies highly involving customers in the design process versus PLM Adoption 


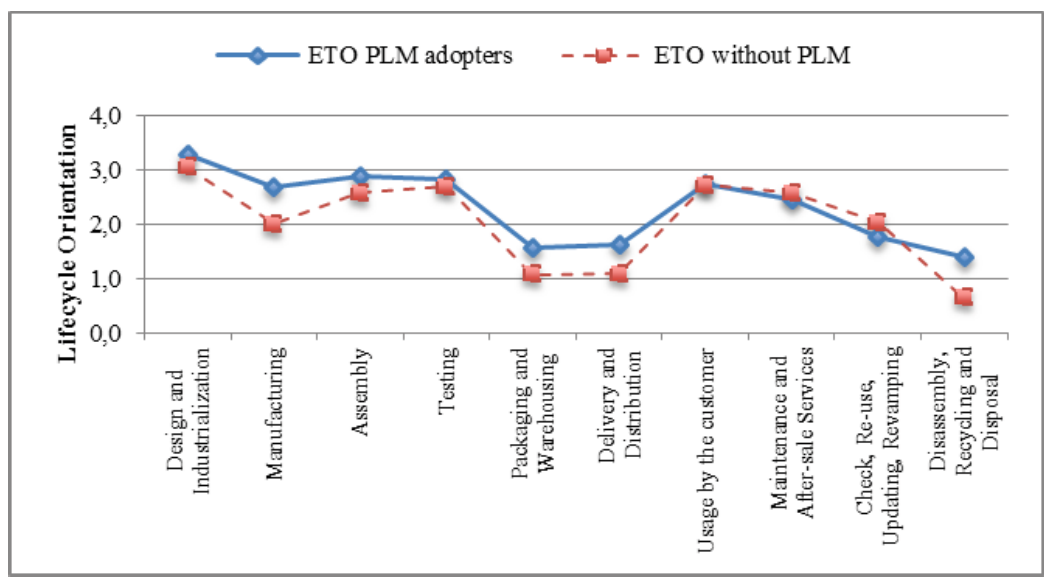

Fig. 7. Lifecycle Orientation of ETO companies versus PLM Adoption

\section{Conclusions}

PLM acronym has a wide definition, intrinsically linked with the usage of IT solutions along the ideal product lifecycle. The fact that the PLM acronym is today used and known is already a good result, after that for years a plethora of acronyms and terms have been proposed by vendors, consultants and academics to address similar issues.

Said this, it might be noticed that the use of PLM acronym is not an easy task. In some way, it is possible to argue that the same PLM acronym could generate a kind of confusion in a listener/reader, due to the double meaning that it embodies. The first meaning concerns with the fact that lifecycle phases might be more and more considered in developing a product, and PLM aims to support this. The second refers to the industrial need of managing efficiently the huge amount of data distributed among different functions and actors and dispersed in a plethora of IT solutions, and again PLM aims to do this. PLM has in itself a kind of dichotomy: it is a concept, and it deals with IT.

In the market, there are some lifecycle-oriented companies, which make their design decisions considering the impacts on the lifecycle of their products (how it will be produced, how it will be used, how it will be maintained, etc.). At the same time, in the market there are companies using IT solutions for defining and managing the lifecycle knowledge of their product (or part of it).

In this paper, we have investigated these two dimensions in real companies: Lifecycle Orientation and PLM Adoption. Data showed how Lifecycle Orientation is a matter of customer-orientation: more a company is focused on the needs of its customer more a company is lifecycle-oriented. The adoption of PLM IT solutions is intrinsically a matter of knowledge management. IT systems support the creation and the re-use of knowledge, permitting a better management of design and development processes. Crossing these two 
dimensions, some interesting results appeared: in average, PLM adopters are more lifecycle-oriented than companies without PLM IT solutions. We cannot say the opposite: not all the lifecycle-oriented companies are also PLM adopters. Figure 8 maps the global Lifecycle Orientation of the sample with the PLM Adoption. The first variable is defined as the total area covered by the curve of Lifecycle Orientation of each specific case (analytically it is the integral of the curve). This figure shows how several companies with a high level of Lifecycle Orientation are still without PLM systems in their practices. There are some clusters of companies, which could be explained in terms of time (not all the lifecycle-oriented companies have already installed a PLM system) and/or in terms of needs (PLM systems are installed for managing product knowledge, without any lifecycle reason).

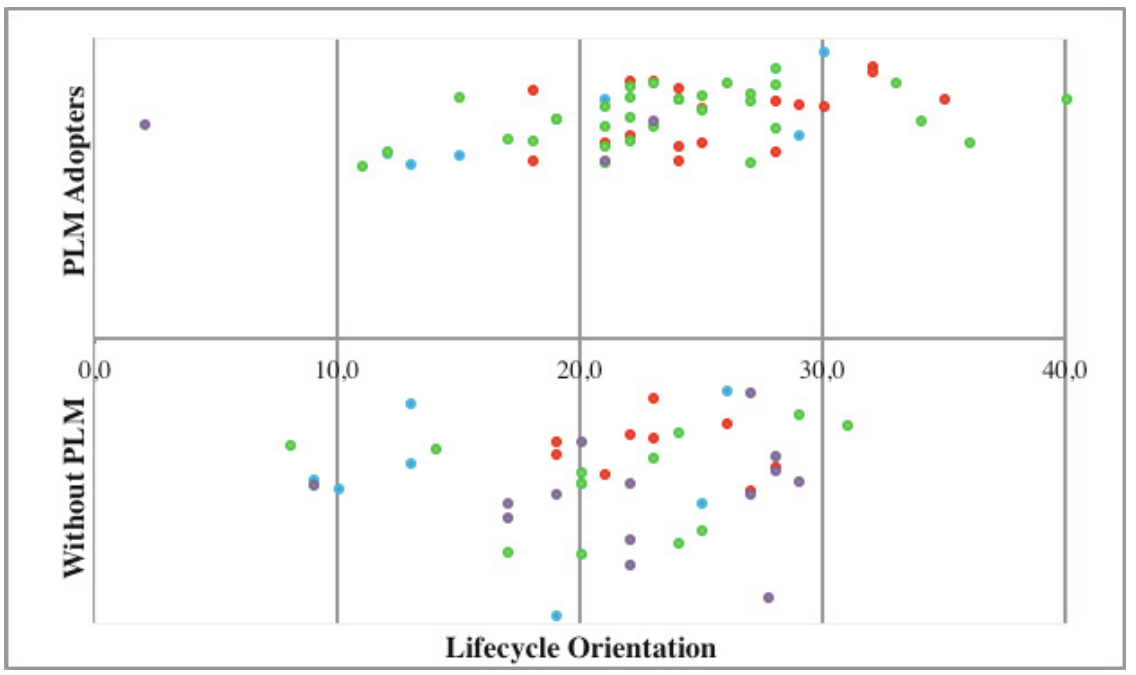

Fig. 8. PLM Adoption and Lifecycle Orientation of the sample (each dot is a company, while the different colors mean companies' dimension)

The performed research has tons of limits. It has been carried on only 103 cases and only in Italy. Not all the performed analysis have been presented, and many of the possible analysis were not performed, not-having adequate data. However, the authors believe that this discussion will arise again in the future of PLM, being intimately part of it.

Acknowledgments. This work was partly funded by the European Commission through the Linked Design Project (FP7-2011-NMP-ICT-FoF, www.linkeddesign.eu). The GeCo Observatory was also partly funded by some PLM vendors. The authors wish to acknowledge their gratitude and appreciation to all the partners and supporters for their contributions. Moreover, the authors wish to thank the reviewers of PLM13 for their valuable comments. 


\section{References}

1. Ameri, F., Dutta, D.: Product Lifecycle Management: Closing the Knowledge Loops. Computer-Aided Design \& Applications 2(5), 577-590 (2005)

2. Garetti, M., Terzi, S., Bertacci, N., Brianza, M.: Organisational change and knowledge management in PLM implementation. Int. J. of Product Lifecycle Management 1(1), 43-51 (2005)

3. GeCo Observatory, http://www. osservatorio-geco.it

4. Rossi, M., Terzi, S., Garetti, M.: Proposal of an Assessment Model for New Product Development. In: Emmanouilidis, C., Taisch, M., Kiritsis, D. (eds.) APMS 2012. IFIP AICT, vol. 397, pp. 383-390. Springer, Heidelberg (2013)

5. Terzi, S., Bouras, A., Dutta, D., Garetti, M., Kiritsis, D.: Product lifecycle management from its history to its new role. Int. J. of Product Lifecycle Management 4(4), 360-389 (2010)

6. Silventoinen, A., Pels, H.J., Kärkkäinen, H., Lampela, H.: Towards future PLM maturity assessment dimensions. In: PLM 2011 - The IFIP WG 5.1 Global Product Development for the Whole Lifecycle, 8th International Conference on Product Lifecycle Management, Eindhoven, The Netherlands, July 11-13 (2011) 